\title{
Aseptic Meningitis as an Initial Manifestation of Anti-myelin Oligodendrocyte Glycoprotein Antibody-associated Disease
}

\author{
Takehiro Suzuki ${ }^{1}$, Kota Maekawa ${ }^{1,2}$, Ko Matsuo $^{1}$, Masayoshi Yamasaki ${ }^{1}$, Masunari Shibata ${ }^{2}$, \\ Toshiyuki Takahashi ${ }^{3,4}$ and Yutaka Naito ${ }^{1}$
}

\begin{abstract}
:
Antibody against myelin oligodendrocyte glycoprotein (MOG-IgG) associated encephalitis is an important syndrome associated with MOG-IgG. However, there have been no reports of MOG-IgG-associated optic neuritis or demyelination following meningitis without encephalitic symptoms. A 55-year-old woman presented to our hospital with headache, nausea, fever, and nuchal rigidity that had persisted for more than a month. She was hospitalized due to aseptic meningitis and recovered with conservative therapy. However, she was re-admitted due to left optic neuritis and demyelinating lesions. We diagnosed MOG-IgG-associated neuromyelitis optica spectrum disorder (NMOSD). She responded to treatment with intravenous methylprednisolone and oral prednisolone. Aseptic meningitis may be an initial manifestation of MOG-IgG-positive NMOSD.
\end{abstract}

Key words: anti-myelin oligodendrocyte glycoprotein antibody, aseptic meningitis, neuromyelitis optica spectrum disorder, optic neuritis

(Intern Med 58: 3319-3321, 2019)

(DOI: 10.2169/internalmedicine.2845-19)

\section{Introduction}

Myelin-oligodendrocyte glycoprotein (MOG) is exclusively expressed on the surface of oligodendrocytes in the central nervous system (CNS). Anti-MOG antibody (MOG$\mathrm{IgG}$ ) is predominantly detected in pediatric acute disseminated encephalomyelitis, recurrent optic neuritis, and aquaporin-4-antibody-seronegative neuromyelitis optica spectrum disorder (NMOSD). MOG-IgG-associated encephalitis is an important clinical syndrome associated with MOG-IgG and may be a bridging element in the emerging spectrum of MOG-IgG-associated syndrome (1). However, there have been no reports of MOG-IgG-associated meningitis without encephalitic symptoms followed by optic neuritis or demyelination.

We herein report a case of MOG-IgG associated NMOSD in a patient with prolonged meningeal symptoms without any encephalitic manifestations for 2 months before the development of optic neuritis and demyelinating lesions.

\section{Case Report}

A 55-year-old woman visited our hospital complaining of headache, nausea, vomiting, and fever, which had persisted for more than one month; with no recent history of illness or drug use and was alert. The patient had no focal neurological deficits, with the exception of nuchal rigidity. A peripheral blood smear revealed leukocytosis (white blood cell count; 9,900/ $\mu \mathrm{L}$ ). The cerebrospinal fluid (CSF) pressure was $25 \mathrm{~cm}$ of $\mathrm{H}_{2} \mathrm{O}$, the cell count was $190 / \mathrm{mm}^{3}$ (monocytes, $127 / \mathrm{mm}^{3}$ ), and the protein level was $78 \mathrm{mg} / \mathrm{dL}$. Magnetic resonance imaging (MRI) revealed hyperintensity without edema in the superficial cortex on fluid-attenuated inversion

${ }^{1}$ Department of Neurology, Ise Red Cross Hospital, Japan, ${ }^{2}$ Department of Neuroendovascular Therapy, Ise Red Cross Hospital, Japan, ${ }^{3}$ Department of Neurology, Tohoku University Graduate School of Medicine, Japan and ${ }^{4}$ Department of Neurology, National Hospital Organization Yonezawa Hospital, Japan

Received: February 18, 2019; Accepted: June 10, 2019; Advance Publication by J-STAGE: July 22, 2019

Correspondence to Dr. Kota Maekawa, k_mae21@kuhp.kyoto-u.ac.jp 

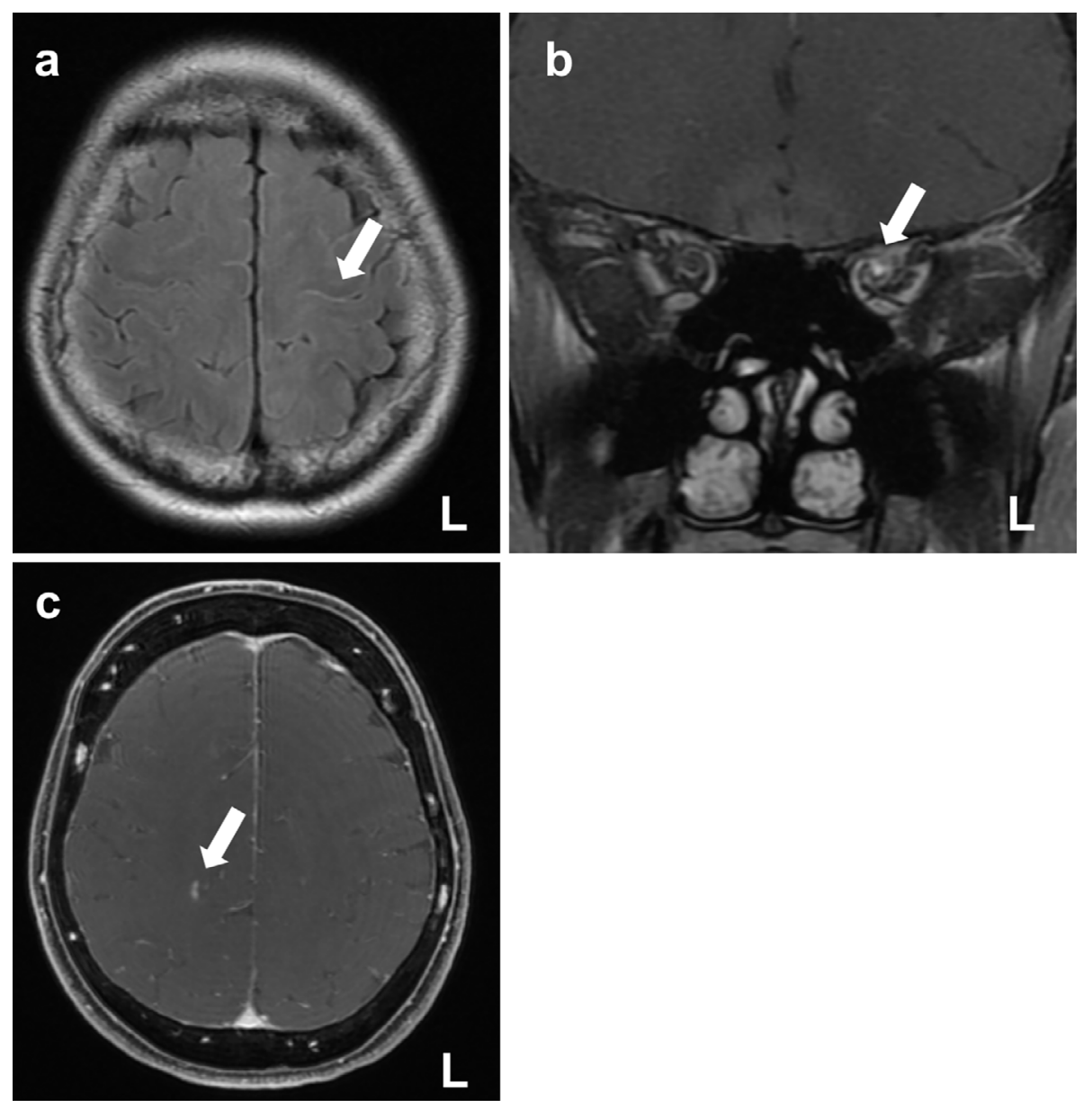

Figure. Head MRI at first and second admission. Hyperintensity was detected in the superficial cortex on FLAIR at the first admission (a). Brain contrast MRI with gadolinium enhancement showed left optic nerve and right optic tract swelling in FLAIR and T1-weighted images on second admission (b). Demyelination lesions were observed in the left prefrontal and right prefrontal-parietal subcortex (c). MRI: magnetic resonance imaging, FLAIR: fluid-attenuated inversion recovery

recovery (FLAIR) images (Figure a). No bacteria or fungi were detected in the patient's blood or CSF. Additionally, the interferon-gamma release assay for tuberculosis was negative. The patient's herpes simplex virus and varicella zoster virus antibody levels were not elevated and the patient was negative for anti-neutrophil cytoplasmic antibodies. The patient was diagnosed with aseptic meningitis and hospitalized for treatment. The patient received conservative therapy and by day 6 of hospitalization, her CSF cell count was $60 / \mathrm{mm}^{3}$ (monocyte; $51 / \mathrm{mm}^{3}$ ) and her protein level was $50 \mathrm{mg} / \mathrm{dL}$. The patient recovered and was discharged on the 13th day after admission.

At seven days after her initial discharge, the patient was re-admitted to our hospital due to severe left-eye visual impairment and loss of direct light reflex. The patient's CSF cell count was $26 / \mathrm{mm}^{3}$ (monocyte; $25 / \mathrm{mm}^{3}$ ) and her protein level was $35 \mathrm{mg} / \mathrm{dL}$, which was better than previously recorded. MRI with gadolinium enhancement revealed left optic nerve and right optic tract swelling on FLAIR and T1weighted imaging (Figure b). Moreover, hyperintensity was observed in the left prefrontal and right prefronto-parietal subcortex (Figure c). The patient was negative for autoanti- bodies, including anti-aquaporin-4 antibodies; however, an in-house live cell-based assay was positive for MOG-IgG. The patient's myelin basic protein (MBP) level was normal and oligoclonal IgG bands were evident.

We diagnosed the patient with MOG-IgG associated NMOSD and initiated treatment with methylprednisolone (1,000 mg/day for 3 days) followed by oral prednisolone (45 mg/kg). The patient's symptoms and MRI gradually improved without any side effects. After continuing oral prednisolone ( $30 \mathrm{mg} /$ day), the patient was discharged on the 39 th day after the 2nd admission.

\section{Discussion}

In the present case, prolonged meningeal symptoms occurred for 2 months. No neurological deficit was observed and the meningeal sign was evident. MOG-IgG was not measured at this time. The patient's symptoms gradually improved with conservative therapy; however, MOG-IgGpositive optic neuritis and demyelinating lesions were diagnosed later. Akaishi et al. reported that optic neuritis, encephalitis, and myelitis were more frequently present in 
MOG-IgG-associated diseases (2). This case was a rare type of MOG-IgG-associated disorder in adults presenting as meningitis and optic neuritis.

While the association of MOG-IgG with optic neuritis and myelitis is now well established, very less is known about the extra-opticospinal manifestations of MOG-IgGrelated autoimmunity $(3,4)$. Previous studies have reported rare cases of MOG-IgG-associated encephalitis $(1,5,6)$. However, in the present case, encephalitic symptoms such as seizures, consciousness disturbance, and focal signs were not seen, and thus the patient was diagnosed with meningitis. To the best of our knowledge, there have been no reports of optic neuritis or demyelination after meningitis. Narayan et al. reported two cases, wherein MOG-IgG-related syndrome presented with symptoms of meningitis and optic neuritis (7). However, their symptoms occurred at the same time, whereas meningitis preceded optic neuritis by more than one month in our patient. We therefore report the first case in which no symptoms other than aseptic meningitis were evident prior to MOG-IgG positive NMOSD. Ogawa et al. suggested that there is a possibility in which MOG-IgG itself may not be directly associated with cerebral cortical encephalitis with epileptic seizures and that another autoimmune disorder involving an unknown autoantibody may coexist with MOG-IgG positivity and might be responsible for encephalitis (5). Thus, in our patient, MOG-IgG and other autoantibodies may have been associated with the aseptic meningitis preceding optic neuritis and demyelination (other autoantibodies were not measured). We conclude that our case and various other previous cases showed MOG-IgGassociated encephalomeningitis and that the primary difference between these cases was the degree of inflammation. In this case, the FLAIR signal increased with enhancement of the subcortex, which means that she had encephalitis at that time. Because of the lower degree of severity, the symptoms of encephalitis were not evident. A preceding infectious prodrome has been reported in $47 \%$ of MOG-IgG positive cases (6). The role of infectious agents in triggering MOG-IgG production is still unclear. It is possible that the patient's aseptic meningitis was infectious meningitis preceding optic neuritis and demyelination; however, bacterial, fungal, herpes-viral, and drug-induced meningitis were excluded at that time. We did not investigate the possibility of enterovirus infection. Although enterovirus meningitis usually improves within 7-10 days, in our patient meningeal symptoms persisted for approximately two months and the clinical course was atypical for viral meningitis (8). Prior infection with other types of viral meningitis unrelated to MOG-IgG could not be denied in this case because MOGIgG was not measured previously; neither electroencephalography nor single positron emission computed tomography was advised at the first onset of meningitis. On the other hand, the long duration of the symptoms of meningitis and the hyperintensity of the superficial cortex on FLAIR may be pathophysiologically similar to the findings in the previous study of MOG-IgG-associated cerebral cortical encephalopathy (5). Although the normal CSF-MBP level indicates that the demyelination was mild, the evident oligoclonal $\mathrm{IgG}$ band showed chronic inflammation of the CNS, which is indicative of possible continuous meningitis. Thus, it is possible that the aseptic meningitis in our patient was associated with autoantibodies and not infection.

\section{Conclusion}

Aseptic meningitis may be an initial manifestation of MOG-IgG-positive NMOSD. MOG-IgG may be partially associated with benign aseptic meningitis that persists for longer than usual. In order to validate these results, further studies should be performed to investigate the results of autoantibody screening tests in patients with aseptic meningitis and to assess the effectiveness of MOG-IgG measurement as a prognostic factor.

The authors state that they have no Conflict of Interest (COI).

\section{References}

1. Hamid SHM, Whittam D, Saviour M, et al. Seizures and encephalitis in myelin oligodendrocyte glycoprotein $\mathrm{IgG}$ disease vs aquaporin 4 IgG disease. JAMA Neurol 75: 65-71, 2018.

2. Akaishi T, Sato DK, Takahashi T, Nakashima I. Clinical spectrum of inflammatory central nervous system demyelinating disorders associated with antibodies against myelin oligodendrocyte glycoprotein. Neurochem Int 2018. Forthcoming.

3. Dale RC, Tantsis EM, Merheb V, et al. Antibodies to MOG have a demyelination phenotype and affect oligodendrocyte cytoskeleton. Neurol Neuroimmunol Neuroinflamm 1: e12, 2014.

4. Konig FB, Wildemann B, Nessler S, et al. Persistence of immunopathological and radiological traits in multiple sclerosis. Arch Neurol 65: 1527-1532, 2008.

5. Ogawa R, Nakashima I, Takahashi T, et al. MOG antibodypositive, benign, unilateral, cerebral cortical encephalitis with epilepsy. Neurol Neuroimmunol Neuroinflamm 4: e322, 2017.

6. Dos Passos GR, Oliveira LM, da Costa BK, et al. MOG-IgGassociated optic neuritis, encephalitis, and myelitis: lessons learned from neuromyelitis optica spectrum disorder. Front Neurol 9: 217, 2018.

7. Narayan RN, Wang C, Sguigna P, Husari K, Greenberg B. Atypical anti-MOG syndrome with aseptic meningoencephalitis and pseudo tumor cerebra-like presentations. Mult Scler Relat Disord 27: 30-33, 2019.

8. Desmond RA, Accortt NA, Talley L, Villano SA, Soong SJ, Whitley RJ. Enteroviral meningitis: natural history and outcome of pleconaril therapy. Antimicrob Agents Chemother 50: 2409-2414, 2006.

The Internal Medicine is an Open Access journal distributed under the Creative Commons Attribution-NonCommercial-NoDerivatives 4.0 International License. To view the details of this license, please visit (https://creativecommons.org/licenses/ by-nc-nd/4.0/).

(C) 2019 The Japanese Society of Internal Medicine

Intern Med 58: 3319-3321, 2019 